\title{
Generalized Riccati equations for 1-D magnetotelluric impedances over anisotropic conductors Part II: Non-uniform source field model
}

\author{
Svetlana Kováčiková and Josef Pek \\ Geophysical Institute, Acad. Sci. Czech Rep., Boční II/1401, CZ-14131 Prague 4-Spořilov, Czech Republic
}

(Received November 16, 2001; Accepted February 19, 2002)

\begin{abstract}
The Riccati equation approach to the analysis of magnetotelluric impedances in 1-D anisotropic structures is generalized to models with non-uniform source field excitation. The problem is solved in the horizontal wavenumber domain. General Riccati matrix equations for the spectral impedances of the medium are derived and their relation to the standard impedance propagation formulae in layered anisotropic models is discussed. Riccati equations give a full solution for the spectral impedances, comprising both the induction and galvanic mode. For a purely inductive excitation of the field, each wave-number harmonics of the magnetic field is strictly linearly polarized on the surface, and only one half of the spectral impedance tensor can be restored. Both induction and galvanic modes generally exist inside the anisotropic conductor and are coupled. A formal similarity between the Riccati equations for a 1-D anisotropic medium with non-uniform sources and those obtained for 2-D laterally inhomogeneous structures is demonstrated, which indicates a possible way of extending the Riccati impedance/admittance equations to multi-dimensional conductors.
\end{abstract}

\section{Introduction}

In the first part of this paper (Kováčiková and Pek, 2002), we have demonstrated that the Riccati equation approach to studying magnetotelluric (MT) impedances in 1-D anisotropic conductors is a simple and effective alternative to the matrix and impedance propagation methods generally used for this purpose in the plane wave model. We will summarize the main theoretical results of that previous study here for reference purposes:

a) The electromagnetic field generated by a vertically propagating time-harmonic plane wave in a 1-D anisotropic structure is controlled by a coupled system of differential equations

$$
\mathbf{E}^{\prime}=\mathbf{D}^{E H} \mathbf{H}, \quad \mathbf{H}^{\prime}=\mathbf{D}^{H E} \mathbf{E},
$$

where the prime stands for $d / d z, \mathbf{E}$ and $\mathbf{H}$ are the horizontal electric and magnetic field vectors, respectively, and the matrix coefficients in (1) are defined as

$$
\begin{aligned}
& \mathbf{D}^{E H}=i \omega \mu_{0} \mathbf{P}, \quad \mathbf{D}^{H E}=\mathbf{P} \boldsymbol{\Sigma}, \quad \mathbf{P}=\left(\begin{array}{cc}
0 & 1 \\
-1 & 0
\end{array}\right), \\
& \boldsymbol{\Sigma}=\left(\begin{array}{cc}
\sigma_{x x}-\sigma_{z z}^{-1} \sigma_{x z}^{2} & \sigma_{x y}-\sigma_{z z}^{-1} \sigma_{x z} \sigma_{y z} \\
\sigma_{x y}-\sigma_{z z}^{-1} \sigma_{x z} \sigma_{y z} & \sigma_{y y}-\sigma_{z z}^{-1} \sigma_{y z}^{2}
\end{array}\right),
\end{aligned}
$$

with $\omega$ being the circular frequency of the field, and $\sigma_{\lambda \nu}, \lambda, v \in\{x, y, z\}$, elements of the conductivity tensor $\boldsymbol{\Sigma}(z)$.

Copy right(C) The Society of Geomagnetism and Earth, Planetary and Space Sciences (SGEPSS); The Seismological Society of Japan; The Volcanological Society of Japan; The Geodetic Society of Japan; The Japanese Society for Planetary Sciences. b) In the plane wave model, no vertical currents and no vertical magnetic fields arise within the 1-D anisotropic structure, i.e. $J_{z}=0$ and $H_{z}=0$ throughout the model.

c) By combining Eqs. (1) and the impedance relation $\mathbf{E}=$ $\mathbf{Z H}$, the generalized Riccati matrix equation for the impedance tensor in a 1-D anisotropic medium is easily obtained,

$$
\mathbf{Z}^{\prime}+\mathbf{Z} \mathbf{D}^{H E} \mathbf{Z}=\mathbf{D}^{E H}
$$

Starting from the homogeneous anisotropic basement of the model, where the impedances are given by simple explicit formulae, the above equation can be easily solved numerically by using any suitable ordinary differential equation solver to give the vertical impedance distribution for any piecewise continuous anisotropic 1-D conductivity section.

So far, we have assumed that the 1-D anisotropic medium is illuminated by a homogeneous plane electromagnetic wave propagating from sources at $z \rightarrow-\infty$ perpendicularly towards the surface of the conductive half-space. In many applications, however, a source, either natural or controlled, of a finite spatial extent has to be considered. In such a case, the primary inducing field is not uniform any more, and the theory developed so far requires some modifications to allow us to manage non-uniform source fields as well.

The interaction of a spatially non-uniform electromagnetic field with an anisotropic medium has been studied in various contexts by many authors for particular source configurations, mainly for dipole source fields in layered conductors (e.g., Chlamtac and Abramovici, 1981; Xiong, 1989; Li and Pedersen, 1992; Everett and Constable, 1999). In these studies, the particular symmetry of the source field 
is exploited explicitly, and closed-form solutions are obtained for the electromagnetic field. From those solutions, analogues of the classical MT transfer functions, i.e. impedances, admittances, or geomagnetic transfer functions, can be found for individual source types.

For general non-uniform source fields and 1-D media, the analysis of the field solution in the horizontal wavenumber domain makes it possible to study the influence of the conductor on the spatial harmonics of the electromagnetic field by means of general spectral transfer functions (e.g., Berdichevsky and Zhdanov, 1984). In this paper we will show that the Riccati equation approach from Kováčiková and Pek (2002) can be readily extended to the spectral impedances in generally anisotropic 1-D conductors.

The structure of the paper is as follows: In Section 2, we present an elementary derivation of the generalized Riccati equations for spectral impedances in a 1-D anisotropic medium, and show their relation to the standard matrix propagation formulae for anisotropic layered structures. A special case of purely inductive excitation, which is relevant for MT and geomagnetic induction studies with very long periods, is discussed in Section 3. Finally, in Section 4, a parallel is drawn between the 1-D anisotropic Riccati equations for a non-uniform source field and generalized Riccati equations for laterally inhomogeneous 2-D conductors.

\section{Riccati Equations for 1-D Anisotropic Media with a Spatially Non-Uniform Primary Excita- tion}

\subsection{Formal derivation of Riccati equations for spectral impedances}

For models with a 1-D horizontally homogeneous distribution of the electrical properties, a solution for the electromagnetic fields is generally sought in the horizontal wavenumber domain. Applying the Fourier transform to Maxwell's equations with a 1-D anisotropic conductivity with respect to $x$ and $y$,

$$
\begin{aligned}
& \mathcal{F}_{x, y}\{\varphi(x, y)\} \equiv \Phi(\xi, \eta) \\
&=\int_{-\infty}^{+\infty} \int_{-\infty}^{+\infty} \varphi(x, y) \\
& \quad \times \exp [-i(\xi x+\eta y)] d x d y,
\end{aligned}
$$

we arrive at a system of differential equations

$$
\begin{aligned}
& i \xi e_{z}-e_{y}^{\prime}=i \omega \mu_{0} h_{x}, \\
& i \xi h_{z}-h_{y}^{\prime}=j_{x}=\sigma_{x x} e_{x}+\sigma_{x y} e_{y}+\sigma_{x z} e_{z}, \\
& e_{x}^{\prime}-i \eta e_{z}=i \omega \mu_{0} h_{y}, \\
& h_{x}^{\prime}-i \eta h_{z}=j_{y}=\sigma_{x y} e_{x}+\sigma_{y y} e_{y}+\sigma_{y z} e_{z}, \\
& i \xi e_{y}-i \eta e_{x}=i \omega \mu_{0} h_{z}, \\
& i \xi h_{y}-i \eta h_{x}=j_{z}=\sigma_{x z} e_{x}+\sigma_{y z} e_{y}+\sigma_{z z} e_{z},
\end{aligned}
$$

where the prime stands for $d / d z$, and the arguments of the field spectra $(\xi, \eta, z, \omega)$ and $z$ for the conductivities have been omitted for brevity. Using (3c) to eliminate the vertical field components from (3a) and (3b), we can write differential relations between the horizontal field components, in matrix notation,

$$
\mathbf{e}^{\prime}=\mathbf{d}^{E E} \mathbf{e}+\mathbf{d}^{E H} \mathbf{h}, \quad \mathbf{h}^{\prime}=\mathbf{d}^{H E} \mathbf{e}+\mathbf{d}^{H H} \mathbf{h},
$$

$$
\begin{aligned}
\text { where } \mathbf{e} & =\left(\begin{array}{l}
e_{x} \\
e_{y}
\end{array}\right), \mathbf{h}=\left(\begin{array}{l}
h_{x} \\
h_{y}
\end{array}\right), \text { and } \\
\mathbf{d}^{E E} & =\frac{1}{\sigma_{z z}}\left(\begin{array}{ll}
-i \xi \sigma_{x z} & -i \xi \sigma_{y z} \\
-i \eta \sigma_{x z} & -i \eta \sigma_{y z}
\end{array}\right), \\
\mathbf{d}^{E H} & =\frac{1}{\sigma_{z z}}\left(\begin{array}{cc}
\xi \eta & i \omega \mu_{0} \sigma_{z z}-\xi^{2} \\
-i \omega \mu_{0} \sigma_{z z}+\eta^{2} & -\xi \eta
\end{array}\right), \\
\mathbf{d}^{H E} & =\frac{1}{i \omega \mu_{0}}\left(\begin{array}{cc}
i \omega \mu_{0} \Sigma_{x y}+\xi \eta & i \omega \mu_{0} \Sigma_{y y}-\xi^{2} \\
-i \omega \mu_{0} \Sigma_{x x}+\eta^{2} & -i \omega \mu_{0} \Sigma_{x y}-\xi \eta
\end{array}\right), \\
\mathbf{d}^{H H} & =\frac{1}{\sigma_{z z}}\left(\begin{array}{cc}
-i \eta \sigma_{y z} & i \xi \sigma_{y z} \\
i \eta \sigma_{x z} & -i \xi \sigma_{x z}
\end{array}\right) .
\end{aligned}
$$

In the special case of only the azimuthal anisotropy, i.e., if $\sigma_{x z}=\sigma_{y z}=0$, both matrices $\mathbf{d}^{E E}$ and $\mathbf{d}^{H H}$ are zero, and (4) formally reduces to a form analogous to (1). Nevertheless, even in this simplified case, vertical magnetic fields and vertical currents exist within the medium by virtue of (3c), which is an essential difference as compared with the uniform source model.

For a non-uniform source field, it is generally not possible to express the relation between the horizontal electric and magnetic fields by means of a single impedance formula $\mathbf{E}=\mathbf{Z H}$. This type of relation can be, however, assumed to apply to the individual $(\xi, \eta)$-harmonics of the electromagnetic field, i.e., $\mathbf{e}=\mathbf{z h}$ for each pair of the wave numbers $(\xi, \eta)$. The $2 \times 2$ tensor $\mathbf{z}(\xi, \eta, z, \omega)$ is the spectral impedance of the model (Berdichevsky and Zhdanov, 1984).

By using in (4) the spectral impedance relation $\mathbf{e}=\mathbf{z h}$, we easily obtain

$$
\begin{aligned}
\mathbf{e}^{\prime} & =\mathbf{z}^{\prime} \mathbf{h}+\mathbf{z h}^{\prime} \\
& =\left(\mathbf{z}^{\prime}+\mathbf{z} \mathbf{d}^{H E} \mathbf{z}+\mathbf{z d} \mathbf{d}^{H H}\right) \mathbf{h}=\left(\mathbf{d}^{E E} \mathbf{z}+\mathbf{d}^{E H}\right) \mathbf{h},
\end{aligned}
$$

and, consequently,

$$
\mathbf{z}^{\prime}+\mathbf{z d}^{H E} \mathbf{z}+\left(\mathbf{z d} \mathbf{d}^{H H}-\mathbf{d}^{E E} \mathbf{z}\right)=\mathbf{d}^{E H},
$$

which is a vector Riccati equation for the spectral impedances in a 1-D anisotropic medium. When compared to (2), Eq. (5) contains two more terms, resulting from dipping (or slanting) anisotropy, given by the conductivity elements $\sigma_{x z}$ and $\sigma_{y z}$. For $\xi=\eta=0$, Eq. (5) simply reduces to the uniform source equation (2).

Employing (5) in practical evaluations of the spectral impedances requires us to provide a starting value of the impedance at some depth level as an initial condition. Similarly as in the case of the uniform source field, we can initialize the solution of (5) by the spectral impedance tensor at the top, or anywhere inside the homogeneous basement of the model. To obtain this starting impedance explicitly, we will first analyze the field solution of the system (4) for a stratified medium. 
2.2 Field solution in an anisotropic layered medium excited by a non-uniform source field

To find the solution of (4) for the field components within a 1-D anisotropic medium that consists of a stack of homogeneous layers underlain by a homogeneous anisotropic half-space, we could almost exactly duplicate the procedure for solving (1) with the uniform source field (see Kováčiková and Pek, 2002, section 2.2).

Let us assume that the solution of (4) is of an exponential form, $e_{v}=e_{0 v} \exp (k z), h_{v}=h_{0 v} \exp (k z), v \in\{x, y\}$, for a given spatial wave number pair $(\xi, \eta)$. Using these expressions in (4), we obtain a homogeneous system of four linear equations for the amplitudes $e_{0 v}$ and $h_{0 v}, v \in\{x, y\}$. For this system to give a non-zero solution for the field amplitudes its determinant has to be zero, which leads to

$$
\operatorname{det}\left(\begin{array}{cc}
\mathbf{d}^{E E}-k \mathbf{1} & \mathbf{d}^{E H} \\
\mathbf{d}^{H E} & \mathbf{d}^{H H}-k \mathbf{1}
\end{array}\right)=0
$$

with 1 being a $2 \times 2$ unit matrix. By evaluating the above determinant, we arrive at a fourth-order algebraic equation for admissible wave numbers $k$,

$$
k^{4}+c_{3} k^{3}+c_{2} k^{2}+c_{1} k+c_{0}=0,
$$

with the coefficients given by

$$
\begin{aligned}
c_{3}= & i\left(\xi \frac{\sigma_{x z}}{\sigma_{z z}}+\eta \frac{\sigma_{y z}}{\sigma_{z z}}\right), \\
c_{2}= & i \omega \mu_{0}\left(\Sigma_{x x}+\Sigma_{y y}\right)-\left(\xi^{2}+\eta^{2}\right) \\
& -\left(\xi^{2} \frac{\sigma_{x x}}{\sigma_{z z}}+\eta^{2} \frac{\sigma_{y y}}{\sigma_{z z}}+2 \xi \eta \frac{\sigma_{x y}}{\sigma_{z z}}\right), \\
c_{1}= & -2 \omega \mu_{0}\left[\left(\xi \sigma_{y y}-\eta \sigma_{x y}\right) \frac{\sigma_{x z}}{\sigma_{z z}}+\left(\eta \sigma_{x x}-\xi \sigma_{x y}\right) \frac{\sigma_{y z}}{\sigma_{z z}}\right] \\
& -2 i\left(\xi^{2}+\eta^{2}\right)\left(\xi \frac{\sigma_{x z}}{\sigma_{z z}}+\eta \frac{\sigma_{y z}}{\sigma_{z z}}\right), \\
c_{0}= & \left(\xi^{2}+\eta^{2}\right)\left(\xi^{2} \frac{\sigma_{x x}}{\sigma_{z z}}+\eta^{2} \frac{\sigma_{y y}}{\sigma_{z z}}+2 \xi \eta \frac{\sigma_{x y}}{\sigma_{z z}}\right) \\
& -\omega^{2} \mu_{0}^{2} \frac{\operatorname{det} \sigma}{\sigma_{z z}}-i \omega \mu_{0}\left(\xi^{2} \Sigma_{x x}+\eta^{2} \Sigma_{y y}+2 \xi \eta \Sigma_{x y}\right) \\
& -i \omega \mu_{0}\left(\xi^{2}+\eta^{2}\right) \frac{\operatorname{det} \sigma_{h}}{\sigma_{z z}},
\end{aligned}
$$

where $\boldsymbol{\sigma}_{h}=\left(\begin{array}{ll}\sigma_{x x} & \sigma_{x y} \\ \sigma_{x y} & \sigma_{y y}\end{array}\right)$.

Clearly, for the case of a purely azimuthal anisotropy, i.e., if $\sigma_{x z}=\sigma_{y z}=0$, the coefficients $c_{1}$ and $c_{3}$ are zero, and (6) gives two pairs of wave numbers which differ only by their signs, $\pm k_{1}$ and $\pm k_{2}$. Further solution of (4) for the field components is, in this case, exactly the same as that for the uniform source.

The above symmetry of the wave numbers $k$ is not valid any more for the general case of anisotropy, and Eq. (6) gives four different wave numbers which correspond to four wave modes that can propagate within an anisotropic layer for the given pair of $(\xi, \eta)$. Since $c_{3}$ is purely imaginary, the sum of real parts of the four wave numbers $k$ is always equal to zero, $\sum_{i=1}^{4} \operatorname{Re} k_{i}=0$. We have not yet been able to find a rigorous proof of the relation $\prod_{i=1}^{4} \operatorname{Re} k_{i}>0$, which would mean that there are, though not symmetric any more, still two down-going and two up-going wave modes in the anisotropic layer. Nevertheless, all numerical calculations support this hypothesis.

In Fig. 1, we illustrate the dependence of the wave numbers $k$ on the horizontal wave number $\xi$ and on one of the parameters of the anisotropy, here on the anisotropy $\operatorname{dip} \alpha_{D}$ in particular. The anisotropy parameters are defined via a factorization of the symmetric conductivity tensor $\boldsymbol{\sigma}$ in terms of three principal conductivities, $\sigma_{1}, \sigma_{2}, \sigma_{3}$, and three elementary Euler rotations, $\alpha_{S}, \alpha_{D}, \alpha_{L}$,

$$
\begin{aligned}
\boldsymbol{\sigma}= & \mathbf{R}_{z}^{T}\left(\alpha_{S}\right) \mathbf{R}_{x}^{T}\left(\alpha_{D}\right) \mathbf{R}_{z}^{T}\left(\alpha_{L}\right) \\
& \times \operatorname{diag}\left\{\sigma_{1}, \sigma_{2}, \sigma_{3}\right\} \mathbf{R}_{z}\left(\alpha_{L}\right) \mathbf{R}_{x}\left(\alpha_{D}\right) \mathbf{R}_{z}\left(\alpha_{S}\right),
\end{aligned}
$$

where $\mathbf{R}_{a}\left(\alpha_{A}\right)$ is the matrix of an elementary rotation by $\alpha_{A}$ around the axis $a$. For schematic dyke models of anisotropic structures, the angles $\alpha_{S}, \alpha_{D}$ and $\alpha_{L}$ can be identified with physically transparent directions of the anisotropy strike, dip and slant, respectively.

The $\xi$-dependence of $k$ (Fig. 1, top) is plotted within the range of $\xi \in\left(10^{-8}, 10^{-5}\right) \mathrm{m}^{-1}$, which roughly corresponds to the horizontal wave lengths from an almost uniform field $\left(6 \times 10^{5} \mathrm{~km}\right)$ down to about $600 \mathrm{~km}$ for a period of $1000 \mathrm{~s}$. For small values of $\xi$, both the real and imaginary parts of the $k$ 's are close to the corresponding uniform field wave numbers $\left( \pm 1.5432 \times 10^{-6} \times(1-i)\right.$ and $\pm 2.8515 \times 10^{-6} \times(1-i)$, in $\mathrm{m}^{-1}$, in the particular setting used here). With increasing $\xi$, the positive and negative branches of the real parts of the wave numbers $k$ start to diverge and approach $\pm \infty$ asymptotically. Only slight asymmetry can be detected in the real parts of $k$, which is a feature observed for even much larger contrasts between the principal resistivities. With increasing $\xi$, the imaginary parts of $k$ show much larger asymmetry, which depends greatly on the particular values of the non-azimuthal elements of the conductivity tensor $\sigma_{x z}, \sigma_{y z}$. The plots in the bottom panels of Fig. 1 then pick out one particular point from the previous plots, specifically $\xi=2 \pi \times 10^{-6} \mathrm{~m}^{-1}$, and show the dependence of $k$ on the anisotropy $\operatorname{dip} \alpha_{D} \in\left(-90^{\circ}, 90^{\circ}\right)$.

To find the solution to (4) for the field vectors $\mathbf{e}$ and $\mathbf{h}$ for a particular pair of the spatial frequencies $(\xi, \eta)$, we can again adopt the matrix propagation procedure used earlier for the plane wave model (Kováčiková and Pek, 2002, section 2.2). The principal difference with respect to the uniform field case is the general presence of both the vertical magnetic fields and vertical currents within the structure for non-zero $\xi$ and $\eta$. From (3c) we can see that the vertical magnetic field is given by a projection of the horizontal electric vector e onto the direction defined by the vector $\mathbf{V}^{T}=(-i \eta, i \xi)$, i.e., $h_{z}=\left(i \omega \mu_{0}\right)^{-1} \mathbf{V}^{T}$ e. Similarly, $j_{z}=\mathbf{V}^{T} \mathbf{h}$. It will show to be advantageous for the solution of (4) to change to a new coordinate system defined by orthogonal vectors $\mathbf{U}^{T}=(i \xi, i \eta)$ and $\mathbf{V}^{T}$, specified above, or into a related (real) orthonormal base given by

$$
\mathbf{u}^{T}=\frac{1}{\sqrt{\xi^{2}+\eta^{2}}}(\xi, \eta), \quad \mathbf{v}^{T}=\frac{1}{\sqrt{\xi^{2}+\eta^{2}}}(-\eta, \xi) .
$$

By virtue of $\nabla \cdot \mathbf{H}=0$ and $\nabla \cdot \mathbf{J}=0$, the vector $\mathbf{U}$ represents a factor relating the vertical change of the vertical 

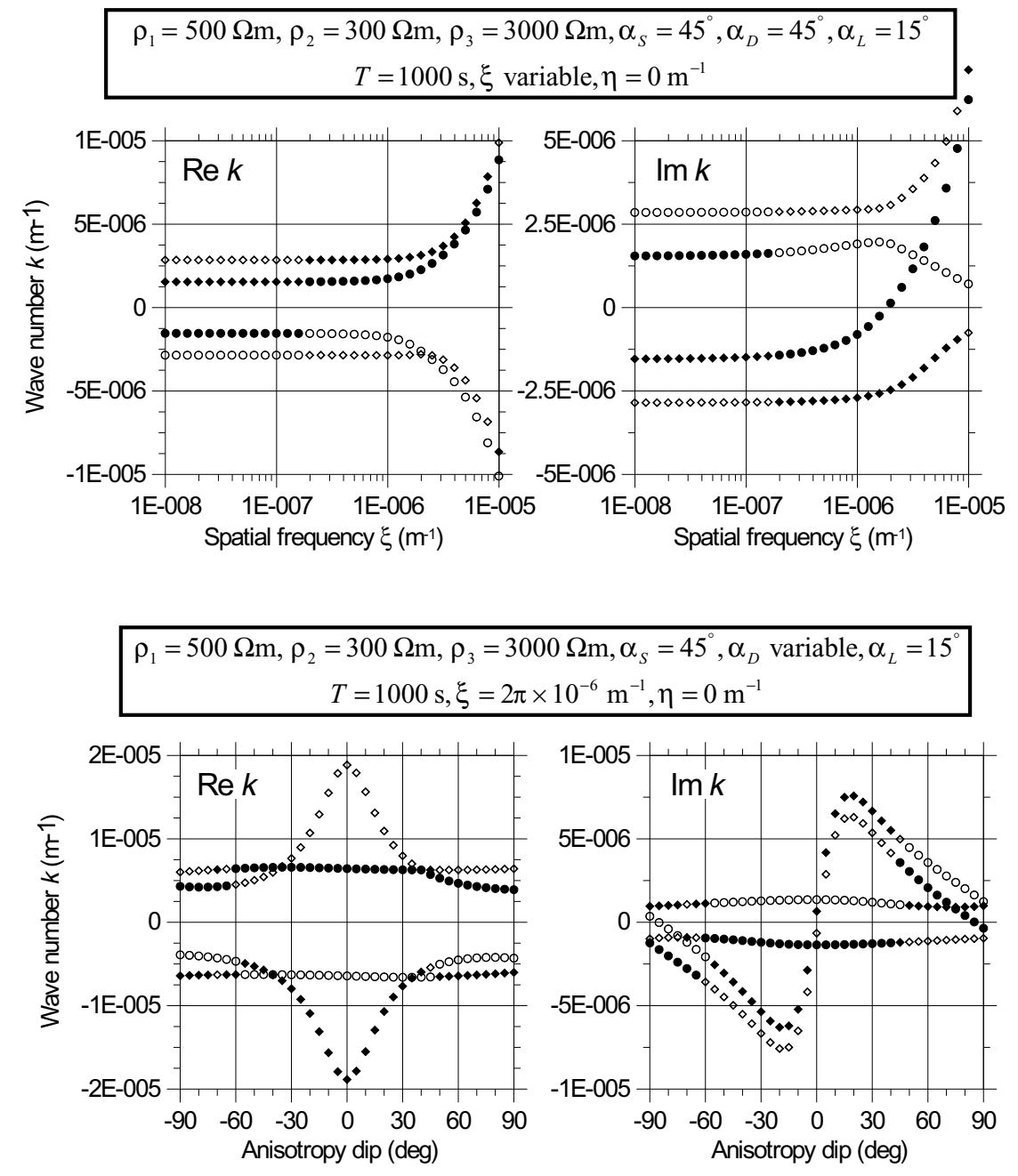

Fig. 1. Real and imaginary parts of the four roots $k$ of the normal Eq. (6) as a function of the horizontal spatial frequency $\xi$ for fixed anisotropy parameters (top panels) and of the anisotropy dip for a fixed pair of spatial frequencies (bottom panels). The corresponding model paramters are listed at the top of the panels. The four symbols, full and empty circles and full and empty diamonds, correspond to the four roots of (6) found by the numerical procedure DZPOCC (Jenkins-Traub three-stage algorithm for finding the zeros of a polynomial, double precision version) from the IMSL library package. The roots are given in the original order provided by the numerical algorithm so that the individual branches of $k$ may consist of various symbols. Notice the scale change for the imaginary parts.

magnetic fields and currents to the horizontal fields in the $(\xi, \eta)$-domain, $h_{z}^{\prime}=-\mathbf{U}^{T} \mathbf{h}$ and $j_{z}^{\prime}=-\mathbf{U}^{T} \mathbf{j}$.

The transition to the $(\mathbf{u}, \mathbf{v})$-based coordinate system is a simple rotation in the horizontal plane by $\varphi=$ $\arccos \left(\xi / \sqrt{\xi^{2}+\eta^{2}}\right)$. By this rotation, Eqs. (4) transform to

$$
\begin{aligned}
\mathbf{u}^{T} \mathbf{e}^{\prime} \equiv e_{u}^{\prime}= & -\tau_{u z} e_{u}-\tau_{v z} e_{v} \\
& +\left(i \omega \mu_{0}-\frac{\xi^{2}+\eta^{2}}{\sigma_{z z}}\right) h_{v} \\
\mathbf{v}^{T} \mathbf{h}^{\prime} \equiv h_{v}^{\prime}= & -\sigma_{u u} e_{u}-\sigma_{u v} e_{v}-\tau_{u z} h_{v} \\
\mathbf{v}^{T} \mathbf{e}^{\prime} \equiv e_{v}^{\prime}= & -i \omega \mu_{0} h_{u} \\
\mathbf{u}^{T} \mathbf{h}^{\prime} \equiv h_{u}^{\prime}= & \sigma_{u v} e_{u}+\left(\sigma_{v v}-\frac{\xi^{2}+\eta^{2}}{i \omega \mu_{0}}\right) e_{v}+\tau_{v z} h_{v}
\end{aligned}
$$

where the following notation has been introduced:

$$
\tau_{u z}=i\left(\xi \frac{\sigma_{x z}}{\sigma_{z z}}+\eta \frac{\sigma_{y z}}{\sigma_{z z}}\right),
$$

$$
\begin{aligned}
\tau_{v z} & =i\left(-\eta \frac{\sigma_{x z}}{\sigma_{z z}}+\xi \frac{\sigma_{y z}}{\sigma_{z z}}\right) \\
\sigma_{u u} & =\mathbf{u}^{T} \boldsymbol{\Sigma} \mathbf{u}=\frac{1}{\xi^{2}+\eta^{2}}\left(\xi^{2} \Sigma_{x x}+\eta^{2} \Sigma_{y y}+2 \xi \eta \Sigma_{x y}\right), \\
\sigma_{v v} & =\mathbf{v}^{T} \mathbf{\Sigma} \mathbf{v}=\frac{1}{\xi^{2}+\eta^{2}}\left(\eta^{2} \Sigma_{x x}+\xi^{2} \Sigma_{y y}-2 \xi \eta \Sigma_{x y}\right), \\
\sigma_{u v} & =\mathbf{u}^{T} \boldsymbol{\Sigma} \mathbf{v}=\mathbf{v}^{T} \mathbf{\Sigma} \mathbf{u} \\
& =\frac{1}{\xi^{2}+\eta^{2}}\left[\xi \eta\left(\Sigma_{y y}-\Sigma_{x x}\right)+\left(\xi^{2}-\eta^{2}\right) \Sigma_{x y}\right] .
\end{aligned}
$$

This transformation does not affect the wave numbers $k$ for the field modes that can propagate within the layer as given by (6).

Clearly, for an isotropic medium, with $\tau_{u z}=\tau_{v z}=0$ and $\boldsymbol{\Sigma}=\sigma \mathbf{1}$, the system (7) splits into two independent sub-systems, one for $e_{u}$ and $h_{v}$, and the other for $e_{v}$ and $h_{u}$. Since, by virtue of ( $3 \mathrm{c}), h_{v}=-i\left(\xi^{2}+\eta^{2}\right)^{-1 / 2} j_{z}$, the $\left(e_{u}, h_{v}\right)$ mode corresponds to the field generated by vertical currents, which have to be injected into the structure for this mode to arise. This is a galvanic field mode and corresponds to 
the poloidal component of the current density $\mathbf{j}$ and toroidal component of the magnetic field $\mathbf{h}$ (Yin and Weidelt, 1999). The other mode $\left(e_{v}, h_{u}\right)$ does not allow any vertical currents to exist within the structure and describes an inductive excitation of the field in the model. It is an inductive mode and corresponds to the toroidal component of the current density $\mathbf{j}$ and poloidal part of the magnetic field $\mathbf{h}$.

In the case of an anisotropic layer, the galvanic and inductive modes become coupled. For a purely azimuthal anisotropy, the coupling is due to $\sigma_{u v} \neq 0$. In the case of a general anisotropy, the non-zero factors $\tau_{u z}$ and $\tau_{v z}$ make the coupling even more complex. Nonetheless, Eqs. (7) form a system of four first-order linear differential equations for the field components and can be solved by the matrix propagation method in a similar way as system (1), except that there is no longer the possibility of separating the electric and magnetic fields by additionally differentiating the firstorder equations with respect to $z$.

Let us assume the four field modes in an anisotropic layer to be characterized by wave numbers $k_{1 p}, k_{1 n}, k_{2 p}$ and $k_{2 n}$, where the symbols ' $p$ ' and ' $n$ ' distinguish the wave numbers with the positive and negative real parts respectively. Generally, $k_{i p} \neq-k_{i n}, i=1,2$, in the anisotropic medium. In Kováčiková and Pek (2002, eq. (13)), we introduced a transition matrix $\mathbf{T}(h)$ that relates the horizontal fields on the top and the bottom of an anisotropic layer in the plane wave model. The analogy of the transition matrix $\mathbf{T}(h)$ for the non-uniform field, $\mathbf{t}(\xi, \eta, h)$, will now contain all four different wave numbers. By duplicating the procedure leading to $\mathbf{T}(h)$, the matrix $\mathbf{t}(\xi, \eta, h)$ can be shown to be, in the $(u, v)$-coordinate system,

$$
\mathbf{t}(\xi, \eta, h)=\mathbf{m}(\xi, \eta) \mathbf{x}(\xi, \eta,-h) \mathbf{m}^{-1}(\xi, \eta),
$$

where

$$
\begin{aligned}
& \mathbf{m}(\xi, \eta)=\left(\mathbf{c}_{1 p}^{T}\left|\mathbf{c}_{1 n}^{T}\right| \mathbf{c}_{2 p}^{T} \mid \mathbf{c}_{2 n}^{T}\right), \\
& \mathbf{c}_{v}=\left(1, Q_{v}, \gamma k_{v} Q_{v},-\gamma k_{v} R_{v}\right), \\
& v \in\left\{k_{1 p}, k_{1 n}, k_{2 p}, k_{2 n}\right\}, \\
& Q_{v}=-\frac{\left(k_{v}+\tau_{u z}\right)^{2}+\left(i \omega \mu_{0}+\lambda^{2}\right) \sigma_{u u}}{\left(k_{v}+\tau_{u z}\right) \tau_{v z}+\left(i \omega \mu_{0}+\lambda^{2}\right) \sigma_{u v}}, \\
& R_{v}=\frac{i \omega \mu_{0}}{k_{v}} \frac{\left(k_{v}+\tau_{u z}\right) \sigma_{u v}-\tau_{v z} \sigma_{u u}}{\left(k_{v}+\tau_{u z}\right) \tau_{v z}+\left(i \omega \mu_{0}+\lambda^{2}\right) \sigma_{u v}}, \\
& \lambda^{2}=-\frac{\xi^{2}+\eta^{2}}{\sigma_{z z}},
\end{aligned}
$$

and

$$
\begin{array}{r}
\mathbf{x}(\xi, \eta,-h)=\operatorname{diag}\left\{\exp \left(-k_{1 p} h\right), \exp \left(-k_{1 n} h\right),\right. \\
\left.\exp \left(-k_{2 p} h\right), \exp \left(-k_{2 n} h\right)\right\} .
\end{array}
$$

Here, $\gamma=\left(-i \omega \mu_{0}\right)^{-1}$. The relation between the impedances on the bottom and the top of the layer are given for each pair of $(\xi, \eta)$ by

$$
\mathbf{z}_{\mathrm{top}}=\left[\mathbf{t}^{E E} \mathbf{z}_{\mathrm{bot}}+\mathbf{t}^{E H}\right]\left[\mathbf{t}^{H E} \mathbf{z}_{\mathrm{bot}}+\mathbf{t}^{H H}\right]^{-1},
$$

where the factorization of the matrix $\mathbf{t}$ into the four blocks, $\mathbf{t}^{E E}, \mathbf{t}^{E H}, \mathbf{t}^{H E}$, and $\mathbf{t}^{H H}$, is identical to that for $\mathbf{T}$ in Kováčiková and Pek (2002, eq. (14)).
In the homogeneous basement that underlies the layered structure, only the two modes with the negative real part of $k$ can exist for energetic reasons. From those, we can find the explicit formula for the impedance tensor in the basement, in the $(u, v)$ coordinate system,

$$
\begin{aligned}
\mathbf{z}_{\text {base }}= & \frac{i \omega \mu_{0}}{R_{1 n}-\kappa R_{2 n}} \\
& \times\left(\begin{array}{cc}
Q_{2 n}^{-1}\left(R_{2 n} k_{1 n}^{-1}-R_{1 n} k_{2 n}^{-1}\right) & k_{1 n}^{-1}-\kappa k_{2 n}^{-1} \\
\kappa R_{2 n} k_{1 n}^{-1}-R_{1 n} k_{2 n}^{-1} & Q_{1 n}\left(k_{1 n}^{-1}-k_{2 n}^{-1}\right)
\end{array}\right),
\end{aligned}
$$

where $\kappa=Q_{1 n} / Q_{2 n}$. For $\xi=\eta=0$, the factors $R=1$ and $k_{1 n}=-k_{1}, k_{2 n}=-k_{2}$, and (8) reduces to the corresponding impedance for the uniform source field (Kováčiková and Pek, 2002, eq. (17)).

The impedance (8) is constant within the homogeneous basement and does not depend on the particular conductivity distribution in the layers above the half-space. Consequently, it can be taken as an initial condition for the solution of the Riccati equation (5), even in the case of a piecewise smoothly varying conductivity tensor $\boldsymbol{\sigma}(z)$.

To return from the $(\mathbf{u}, \mathbf{v})$-based system back to the original $(x, y)$ coordinate system, a simple similarity transform has to be applied to the $(u, v)$-impedance tensor,

$$
\mathbf{z}^{(x, y)}=\frac{1}{\xi^{2}+\eta^{2}}\left(\begin{array}{cc}
\xi & -\eta \\
\eta & \xi
\end{array}\right) \mathbf{z}^{(u, v)}\left(\begin{array}{cc}
\xi & \eta \\
-\eta & \xi
\end{array}\right) .
$$

Let us emphasize that, due to the independence of $\mathbf{z}_{\text {base }}$ of the vertical coordinate $z$ within the basement, this impedance can be obtained directly by solving the Riccati algebraic equation

$$
\mathbf{z d}^{H E} \mathbf{z}+\left(\mathbf{z d}^{H H}-\mathbf{d}^{E E} \mathbf{z}\right)=\mathbf{d}^{E H},
$$

which immediately follows from (5) for the case of $\mathbf{z}^{\prime}=0$.

\section{Spectral Impedances for the Induction Mode}

The matrix propagation procedure applied to the 1-D anisotropic layered model excited by a non-uniform source field provides a spectral impedance tensor $\mathbf{z}(\xi, \eta, z, \omega)$ that integrates the contributions from both the induction and galvanic modes of the electromagnetic field. In the anisotropic case, the two modes cannot be decoupled at all, so their distinguishing in that case is rather a matter of terminology only. Nonetheless, for MT as well as for controlled source induction methods, the case of a purely inductive excitation of the electromagnetic field within the earth is of primary interest. The above theory shows, however, that describing in full the field induced within a 1-D anisotropic medium requires us to take both the induction and galvanic modes into consideration.

The basic condition that defines the inductive excitation of the medium is that no currents are injected into the structure, neither on the surface nor anywhere inside the conductive medium. By virtue of ( $3 c)$, this condition gives

$$
\begin{array}{r}
j_{z}=i \sqrt{\xi^{2}+\eta^{2}} \mathbf{v}^{T} \mathbf{h}=i \sqrt{\xi^{2}+\eta^{2}} h_{v}=0 \\
\text { if } z=0 .
\end{array}
$$


For the internal points of the medium, the absence of a current injection is automatically satisfied by considering the condition $\nabla \cdot \mathbf{J}=0$. Hence, the magnetic field of inductive sources is linearly polarized in the direction $\mathbf{u}$ on the surface. Consequently, no information on the impedance elements $z_{u v}$ and $z_{v v}$ can be drawn from purely inductive data. By virtue of $h_{v}=0$, the impedance relations on the surface degenerate to

$$
e_{u}=z_{u u} h_{u}, \quad e_{v}=z_{v u} h_{u} .
$$

Since $\mathbf{u}, \mathbf{v}$ depend on the particular spatial frequencies $\xi, \eta$, the $(\mathbf{u}, \mathbf{v})$-based impedances are not ready for use in a Fourier synthesis from the $(\xi, \eta)$-domain into the $(x, y)$ domain. Formally, the degenerate impedance relations (10) could be rotated into the $(x, y)$-system, which would result in an apparently consistent, full impedance tensor. In fact, however, a full impedance tensor can never be determined uniquely, as, by virtue of the induction condition $h_{v}=0$, any arbitrary values can be used instead of $z_{v u}$ and $z_{v v}$, and rotated together with the correct first column of the impedance matrix, without violating the trueness of the impedance relation. In the original $(x, y)$-system, this fact results in all linear relations $\mathbf{e}=\left(\mathbf{z}_{\text {ind }}+\mathbf{s \mathbf { v } ^ { T }}\right) \mathbf{h}$ to be equivalent, and true, for any arbitrary vector $\mathbf{s}$, where $\mathbf{z}_{\text {ind }}$ is the image of $\left(\begin{array}{ll}z_{u u} & 0 \\ z_{v u} & 0\end{array}\right)$ in the $(x, y)$-system, e.g., with a particular choice of

$$
s_{x}^{a}=\frac{\xi}{\eta} \frac{\xi z_{u u}-\eta z_{v u}}{\sqrt{\xi^{2}+\eta^{2}}}, \quad s_{y}^{a}=-\frac{\eta}{\xi} \frac{\eta z_{u u}+\xi z_{v u}}{\sqrt{\xi^{2}+\eta^{2}}},
$$

we can anti-diagonalize the impedance matrix in the $(x, y)$ system. The 'main' impedances in this case can be easily shown to be

$$
z_{x y}^{a}=\frac{\xi}{\eta} z_{u u}-z_{v u}, \quad z_{y x}^{a}=\frac{\eta}{\xi} z_{u u}+z_{v u} .
$$

It is obvious that, in the isotropic case, with the induction and galvanic modes decoupled, $z_{u u}=0$, and $z_{y x}^{a}=-z_{x y}^{a}=$ $z_{v u}$, which resembles a normal impedance structure for layered models with a plane wave source field.

To demonstrate the effect of non-zero horizontal wave numbers on the induction data, we present results of two simple computations in Fig. 2. For a simple 3-layer model, we illustrate two phenomena which are typical of models with non-uniform primary fields. First, contrary to the uniform source case, we now have an explicit dependence of the impedances on the anisotropy dip within the layered structure. In Fig. 2(a), we show MT curves derived from the impedances $z_{u u}$ and $z_{v u}$ for a simple 3-layer model with parameters $h_{1}=10 \mathrm{~km}, h_{2}=30 \mathrm{~km}, \varrho_{1}=$ $\varrho_{3}=1000 \Omega \mathrm{m}$. The second layer is anisotropic with different dips $\alpha_{D 2}$ chosen so that the horizontal conductivity $\varrho_{y 2}=\varrho_{2, \max } \cos ^{2} \alpha_{D 2}+\varrho_{2, \min } \sin ^{2} \alpha_{D 2}$ equals that given by the parameters $\varrho_{2, \max }=1000 \Omega \mathrm{m}, \varrho_{2, \min }=10 \Omega \mathrm{m}$, and $\alpha_{2 D}=60^{\circ}$. The strike parallel resistivity of this layer is $\varrho_{x 2}=10 \Omega \mathrm{m}$ for all models. The parameters of the model are intentionally chosen non-realistic to make the effect visible. The non-uniform source is simulated by considering a spatially harmonic field with the horizontal wave lengths $\lambda_{x}=2 \pi / \xi=1000 \mathrm{~km}$ and $\lambda_{y}=2 \pi / \eta=10000 \mathrm{~km}$ in the $x$ and $y$ directions respectively. While for the uniform source field, the curves would be identical for any combination of the anisotropy parameters that preserves $\varrho_{y 2}$, with the non-uniform source field a slight dependence on $\alpha_{D 2}$ is observed, especially for dips close to $90^{\circ}$ with very large $\varrho_{2, \max } / \varrho_{2, \min }$ ratios involved. An asymptotic decrease of the resistivity curves, and a corresponding increase of the phases observed at long periods is a typical behaviour of the induction curves generated by a non-uniform source field (Berdichevsky and Zhdanov, 1984).

Second, contrary to the isotropic case where the impedances depend on the horizontal wave numbers, solely via the factor $\xi^{2}+\eta^{2}$, in the anisotropic case this dependence is not symmetric any more with respect to $\xi$ and $\eta$. In Fig. 2(b), the dependence of the above induction MT curves on particular values of $\xi$ and $\eta$ is shown, with $\xi^{2}+\eta^{2}=1.01 \times 10^{-6} \mathrm{~m}^{-2}$ being constant for all particular $(\xi, \eta)$-pairs. As different pairs of $\xi, \eta$ lead to impedance computations in coordinate systems with different rotations with respect to the $(x, y)$ system, the minimum model resistivity in the $x$-direction is most distinctly expressed by apparent resistivities obtained for small $\xi / \eta$, i.e., large $\lambda_{x} / \lambda_{y}$ ratios.

Equation for $e_{v}$ in (10) can be also considered as a relation for the spectral geomagnetic transfer functions $w_{u}(\xi, \eta, z, \omega)$ and $w_{v}(\xi, \eta, z, \omega)$. By virtue of (3c), we have

$$
h_{z}=\frac{1}{i \omega \mu_{0}} e_{v}=\frac{1}{i \omega \mu_{0}}\left(z_{v u} h_{u}+z_{v v} h_{v}\right) \equiv w_{u} h_{u}+w_{v} h_{v},
$$

which again gives $h_{z}=w_{u} h_{u}$ on the surface in the induction case. In the 1-D anisotropic case, the geomagnetic transfer function is simply equal to the corresponding spectral impedance $z_{v u}$ scaled by the factor $\left(i \omega \mu_{0}\right)^{-1}$. Contrary to the isotropic case, where the geomagnetic transfer functions and the MT spectral impedances contain exactly the same information (for $\xi \neq 0$ and $\eta \neq 0$ ), in the anisotropic case only the main impedance $z_{v u}$ can be resolved from geomagnetic data. Telluric information is indispensable, however, for determining the secondary impedance $z_{u u}$

In the induction theory for isotropic media, it has been proved that, for non-zero field harmonics, the spectral impedances on the surface can be completely recovered from the ratio of the internal and external part of the magnetic field (Berdichevsky and Zhdanov, 1984), a parameter often used in closely related spherical applications, in particular in the geomagnetic depth sounding. In the air, which we consider a perfect insulator, only the induction mode $\left(e_{v}, h_{u}\right)$ can exist. From $(7 \mathrm{c}, 7 \mathrm{~d})$, with all the conductivities set to zero, we easily obtain the solution for $e_{v}$ and $h_{u}$ in the air as a sum of an up-going (internal) and down-going (external) wave,

$$
\begin{aligned}
e_{v} & =C_{0,1} \exp \left(\lambda_{0} z\right)+C_{0,2} \exp \left(-\lambda_{0} z\right), \\
h_{u} & =-\frac{\lambda_{0}}{i \omega \mu_{0}}\left[C_{0,1} \exp \left(\lambda_{0} z\right)-C_{0,2} \exp \left(-\lambda_{0} z\right)\right], \\
\lambda_{0} & =\sqrt{\xi^{2}+\eta^{2}}
\end{aligned}
$$

with $C_{0,1}, C_{0,2}$ being integration constants. On the surface, the ratio of the internal to the external part of the field is given by $r_{\mathrm{ie}}=h_{u}^{\mathrm{int}} / h_{u}^{\mathrm{ext}}=-C_{0,1} / C_{0,2}$, and its relation to 
a
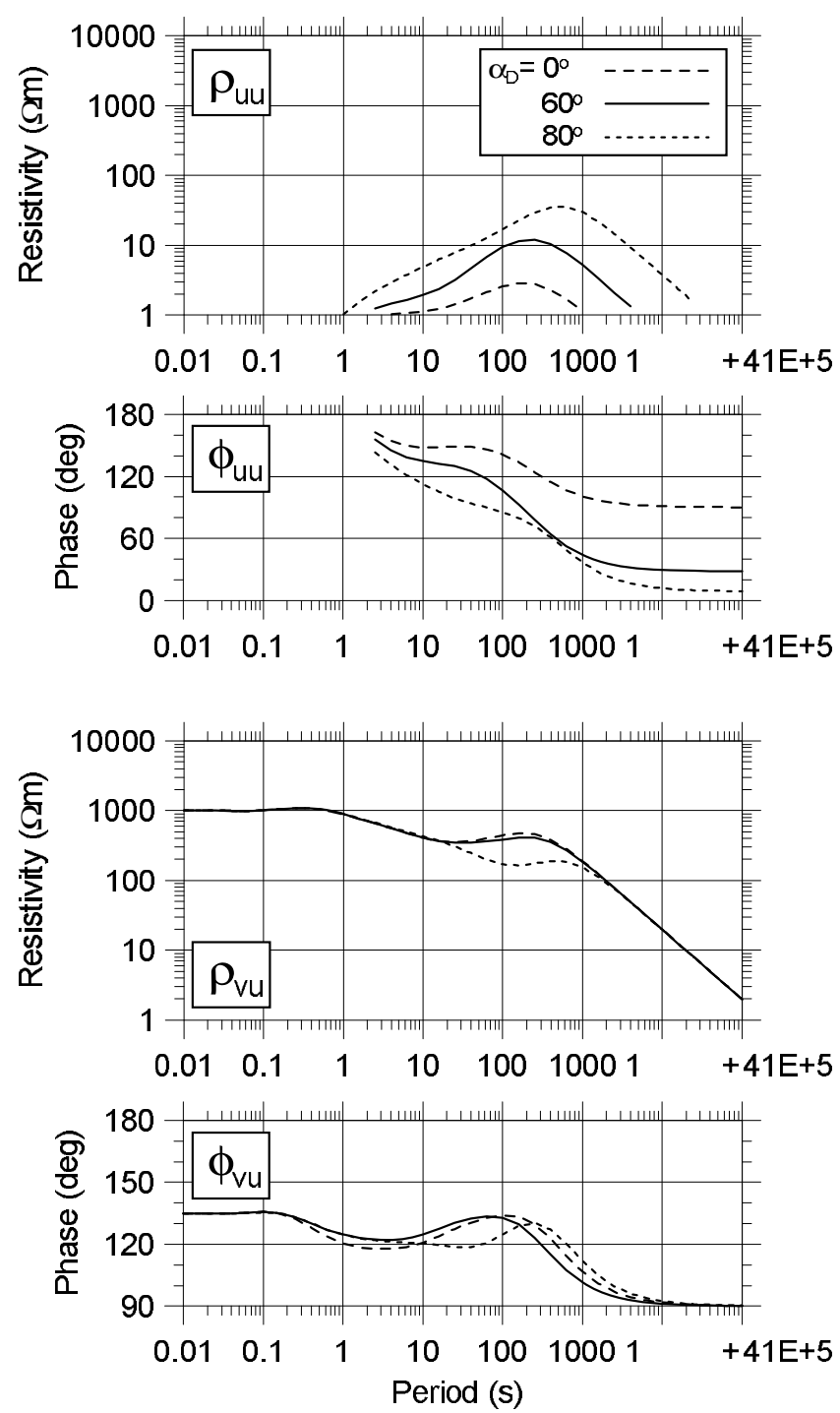

b
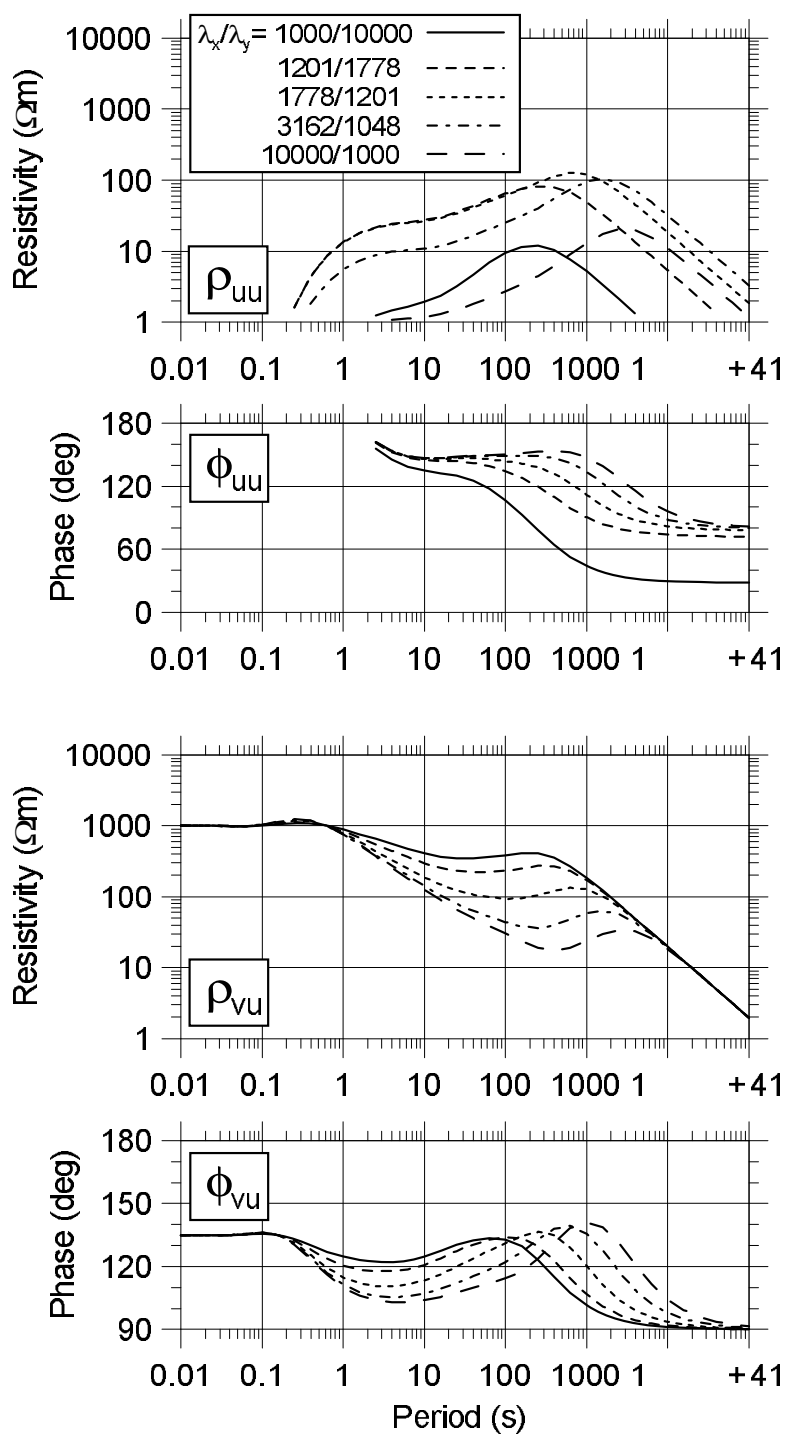

Fig. 2. (a) MT curves of the induction mode for a 3-layer model with dipping anisotropy in the intermediate layer. The model is excited by a non-uniform, horizontally periodic source field. See the text for the model and source field specifications. The anisotropy dip of the second layer varies from 0 to $80^{\circ}$, but the effective horizontal resistivity of the layer is kept constant and equals $257.5 \Omega \mathrm{m}$ for all models. (b) MT curves of the induction mode for the same model, with specifically $\varrho_{2, \max }=1000 \Omega \mathrm{m}, \varrho_{2, \min }=10 \Omega \mathrm{m}$, and $\alpha_{2 D}=60^{\circ}$. The curves are computed for different pairs of horizontal wave numbers $\xi$, $\eta$, with $\xi^{2}+\eta^{2}=$ const $=1.01 \times 10^{-6} \mathrm{~m}^{-2}$. The corresponding horizontal wave lengths, in km, are shown in the inset at the top of each panel.

the spectral impedance is then

$$
r_{\mathrm{ie}}=\frac{i \omega \mu_{0} \lambda_{0}^{-1}+z_{v u}}{i \omega \mu_{0} \lambda_{0}^{-1}-z_{v u}},
$$

or vice versa,

$$
z_{v u}=\frac{i \omega \mu_{0}}{\lambda_{0}} \frac{1+r_{\mathrm{ie}}}{1-r_{\mathrm{ie}}}
$$

Similarly as in the case of the spectral geomagnetic transfer functions, the ratio of the internal and external parts of the magnetic field allows us to recover $z_{v u}$ only, as $z_{u u}$ cannot be found from the magnetic data alone.

\section{1-D and 2-D Riccati Equations}

There is a certain analogy between 1-D models with a non-uniform source field and multi-dimensional models in that the same parameters can be defined to describe the response of the structure, specifically the impedances, geomagnetic transfer functions, and internal/external magnetic field ratios. For a 1-D isotropic model with inductive excitation, those parameters have been proved to be interchangeable as they provide completely equivalent information on the geoelectrical section; specifically, they all result in the impedance $z_{v u}$. For a 1-D anisotropic model, the parameters are only partly equivalent. The secondary impedance $z_{u u}$ cannot be reconstructed from purely magnetic parameters, and telluric information is necessarily required for this purpose. In the multi-dimensional case, the individual parameters become independent functions, each with its own specific way of contributing to the complete geoelectrical characteristics of the model.

The complementary character of the horizontal and verti- 
cal spectral MT transfer functions is also reflected by the corresponding general Riccati equations. For the general 1-D anisotropic case, Eq. (5) can be rewritten in a form explicitly involving the vertical transfer functions

$$
\begin{aligned}
\mathbf{z}^{\prime}+ & \mathbf{z D}{ }^{E H} \mathbf{z}+\left(\mathbf{z d}^{H H}-\mathbf{d}^{E E} \mathbf{z}\right) \\
& +\left(i \omega \mu_{0}\right)^{-1} \mathbf{U} \mathbf{V}^{T} \mathbf{z}-\sigma_{z z}^{-1} \mathbf{U} \mathbf{V}^{T}=\mathbf{D}^{E H} .
\end{aligned}
$$

Here, $\mathbf{D}^{E H}=i \omega \mu_{0} \mathbf{P}$ and $\mathbf{D}^{H E}=\mathbf{P} \boldsymbol{\Sigma}$ are the original, $(\xi, \eta)$-independent matrices encountered in the uniform source theory (see Eq. (1)). By virtue of (3c), we have $h_{z}=\left(i \omega \mu_{0}\right)^{-1} \mathbf{V}^{T} \mathbf{z h}$, and the first extracted term on the 1.h.s. of (11) can be simply considered to be a $(\xi, \eta)$-Fourier image of the $(x, y)$-spatial derivatives of the vertical magnetic transfer function. Similarly, by virtue of $\mathbf{V}^{T} \mathbf{h}=j_{z}$, the second extracted term can be considered to be an image of the horizontal spatial derivatives of a vertical electric transfer function which relates the vertical currents in the structure to the horizontal magnetic field.

The idea of considering, besides the horizontal impedances, also the vertical MT transfer functions, seems to be fruitful when we aim at obtaining analogues of the Riccati impedance equations for the multi-dimensional case. So far, we have succeeded in deriving the Riccati equations for a simple 2-D model without anisotropy, which we will demonstrate here.

Let us consider first the $E$-polarization case for a 2-D model with the strike parallel to $x$, so that only the field components $E_{x}, H_{y}$ and $H_{z}$ exist, related by reduced Maxwell's equations

$$
\begin{aligned}
& \frac{\partial H_{z}}{\partial y}-\frac{\partial H_{y}}{\partial z}=\sigma E_{x}, \\
& \frac{\partial E_{x}}{\partial z}=i \omega \mu_{0} H_{y}, \quad \frac{\partial E_{x}}{\partial y}=-i \omega \mu_{0} H_{z}, \\
& \frac{\partial H_{y}}{\partial y}+\frac{\partial H_{z}}{\partial z}=0 .
\end{aligned}
$$

The conductivity $\sigma=\sigma(y, z)$, and all the fields depend on arguments $y, z$ and $\omega$, which we omit here for brevity. We define the horizontal admittance $Y_{E}$ in a standard way as $Y_{E}=H_{y} / E_{x}$. Additionally, we introduce a vertical admittance by $U_{E}=H_{z} / E_{x}$. Then

$$
\begin{aligned}
\frac{\partial Y_{E}}{\partial z}-\frac{\partial U_{E}}{\partial y} & =\frac{1}{E_{x}}\left(\frac{\partial H_{y}}{\partial z}-\frac{\partial H_{z}}{\partial y}\right)-\frac{H_{y}}{E_{x}^{2}} \frac{\partial E_{x}}{\partial z}+\frac{H_{z}}{E_{x}^{2}} \frac{\partial E_{x}}{\partial y} \\
& =-\sigma-i \omega \mu_{0} Y_{E}^{2}-i \omega \mu_{0} U_{E}^{2},
\end{aligned}
$$

and

$$
\begin{aligned}
\frac{\partial Y_{E}}{\partial y}+\frac{\partial U_{E}}{\partial z}= & \frac{1}{E_{x}}\left(\frac{\partial H_{y}}{\partial y}+\frac{\partial H_{z}}{\partial z}\right) \\
& -\frac{H_{y}}{E_{x}^{2}} \frac{\partial E_{x}}{\partial y}-\frac{H_{z}}{E_{x}^{2}} \frac{\partial E_{x}}{\partial z}=0 .
\end{aligned}
$$

Hence, we have arrived at a complete pair of first-order partial differential equations for the $E$-mode admittances $Y_{E}$ and $U_{E}$,

$$
\begin{aligned}
& \frac{\partial Y_{E}}{\partial z}+i \omega \mu_{0} Y_{E}^{2}-\frac{\partial U_{E}}{\partial y}+i \omega \mu_{0} U_{E}^{2}=-\sigma, \\
& \frac{\partial Y_{E}}{\partial y}+\frac{\partial U_{E}}{\partial z}=0
\end{aligned}
$$

where the first equation consists of a sum of two Riccati operators applied to the respective admittances. The second equation requires that the 2-D admittance field $\left(Y_{E}, U_{E}\right)$ is source-free. Notice that the traditional geomagnetic transfer function, $W_{E}=H_{z} / H_{y}$, is related to the above admittances by $W_{E}=U_{E} / Y_{E}$.

The symmetry of the Riccati equations for admittances and impedances, typical of 1-D media (Kováčiková and Pek, 2002, eq. (1)), does not apply any more to the 2-D case. Rather, a particular type of the Riccati equation is related to a particular field mode, specifically the admittance equation (12) to the $E$-mode, and the impedance equation to the $H$ mode (see Eq. (13) below). That seems to reflect the wellknown conductivity/resistivity asymmetry in the two field modes.

For the 2-D $H$-mode, with only the field components $H_{x}$, $E_{y}$ and $E_{z}$ involved, the governing field equations read

$$
\begin{aligned}
& \frac{\partial E_{z}}{\partial y}-\frac{\partial E_{y}}{\partial z}=i \omega \mu_{0} H_{x}, \\
& \frac{\partial H_{x}}{\partial z}=\sigma E_{y}, \quad \frac{\partial H_{x}}{\partial y}=-\sigma E_{z}, \\
& \frac{\partial\left(\sigma E_{y}\right)}{\partial y}+\frac{\partial\left(\sigma E_{z}\right)}{\partial z}=0 .
\end{aligned}
$$

Similarly as above, we consider the standard horizontal impedance $Z_{H}=E_{y} / H_{x}$, and additionally define a vertical impedance by $V_{H}=E_{z} / H_{x}$. Then

$$
\begin{gathered}
\frac{\partial Z_{H}}{\partial z}-\frac{\partial V_{H}}{\partial y}=\frac{1}{H_{x}}\left(\frac{\partial E_{y}}{\partial z}-\frac{\partial E_{z}}{\partial y}\right)-\frac{E_{y}}{H_{x}^{2}} \frac{\partial H_{x}}{\partial z} \\
+\frac{E_{z}}{H_{x}^{2}} \frac{\partial H_{x}}{\partial y}=-i \omega \mu_{0}-\sigma Z_{H}^{2}-\sigma V_{H}^{2}, \\
\frac{\partial\left(\sigma Z_{H}\right)}{\partial y}+\frac{\partial\left(\sigma V_{H}\right)}{\partial z}=\frac{1}{H_{x}}\left[\frac{\partial\left(\sigma E_{y}\right)}{\partial y}+\frac{\partial\left(\sigma E_{z}\right)}{\partial z}\right] \\
-\frac{\sigma E_{y}}{H_{x}^{2}} \frac{\partial H_{x}}{\partial y}-\frac{\sigma E_{z}}{H_{x}^{2}} \frac{\partial H_{x}}{\partial z}=0 .
\end{gathered}
$$

Consequently, the MT impedances $Z_{H}$ and $V_{H}$ for the 2-D $H$-mode model are described by a system of first-order nonlinear partial differential equations

$$
\begin{aligned}
& \frac{\partial Z_{H}}{\partial z}+\sigma Z_{H}^{2}-\frac{\partial V_{H}}{\partial y}+\sigma V_{H}^{2}=-i \omega \mu_{0}, \\
& \frac{\partial\left(\sigma Z_{H}\right)}{\partial y}+\frac{\partial\left(\sigma V_{H}\right)}{\partial z}=0,
\end{aligned}
$$

where the first equation is a generalized Riccati impedance equation, whereas the second equation represents a constraint that requires the $\sigma$-scaled impedance field $\left(\sigma Z_{H}, \sigma V_{H}\right)$ to be source-free in the 2-D space. It is interesting to notice that on the surface $V_{H}=0$ and, consequently, the Ricatti equation for the $H$-mode reduces to a 1-D Riccati impedance equation with the correct subsurface conductivity $\sigma(y, z=0+)$. Hence, immediately below the surface, a simple 1-D interpretation of the $H$-mode data gives correct conductivities of the structure.

To summarize, in the 2-D isotropic case, the horizontal and vertical admittances, or impedances, are related via Riccati-type 2-D equations and constraining conditions (12) 
and (13). In those equations, the horizontal and vertical MT functions are of equal significance from the point of view of the field description. If the medium degenerates into a 1-D structure, the horizontal and vertical transfer functions can be reduced to a simple impedance form, which is formally expressed by the equivalency of Eqs. (11) and (5).

\section{Conclusion}

The Riccati equation approach to evaluating the MT impedances, or admittances, in a 1-D horizontally homogeneous, generally anisotropic medium can be easily extended to 1-D anisotropic models excited by a non-uniform source field. Large-scale non-uniformity of the source field is a significant factor in high latitute electromagnetic soundings or in regions near to the equator. On a small scale, practically all controlled source methods require consideration of a non-uniform source model.

In the most general case, the MT field of a non-uniform source can be obtained by Fourier synthesizing the field harmonics computed for the given model in the horizontal wave-number domain. Generalized vector Riccati equations (5) apply to the spectral impedances for each pair of horizontal wave numbers $\xi, \eta$. As these equations represent general impedance relations in a 1-D medium, they comprise both the induction and galvanic field modes that can arise in a conductor. While in the isotropic case, these two modes can be separated; for anisotropic models they are always coupled inside the medium.

A particular type of source field can be specified either by defining the surface fields or currents in (3), or by introducing artificial field jumps inside the medium if an internal source is considered (see, e.g., Yin and Weidelt, 1999, for a DC dipole field). Particularly, a purely inductive excitation of a 1-D layered medium leads to a strictly linear polarization of the surface magnetic field for any horizontal wave number pair. Consequently, the full spectral impedance tensor can never be completely recovered from purely induction data. Only two impedance elements, $z_{u u}$ and $z_{v u}$ (10), can be uniquely found in this case. For anisotropic models, these inductive spectral impedances are, however, affected by galvanic fields arising due to the mode coupling inside the medium. Hence, contrary to the plane wave model, certain sensitivity to the vertical conductivity and dipping anisotropy within the section develops if a non-uniform source field is involved. For large-scale field non-uniformity and more or less realistic Earth conductivity anisotropies, however, this dependence seems to be rather weak, and the azimuthal anisotropy is still the most significant factor affecting the spectral impedances.
For anisotropic models with a non-uniform inductive source field, the equivalency of the MT and geomagnetic spectral transfer functions, well-known in applying to isotropic media (Berdichevsky and Zhdanov, 1984), is violated. The geomagnetic spectral transfer functions, as well as ratios of the internal and external magnetic fields, allow us to restore the spectral impedance $z_{v u}$, whereas telluric information is necessarily required for $z_{u u}$. This conclusion readily extends to spherical conductors for application in geomagnetic depth sounding. The Riccati equation approach can be simply generalized to the spherical conductors, too, as shown by Eckhardt (1963) for the isotropic earth.

Finally, an analysis of the 1-D Riccati equations for nonuniform source fields seems to indicate a way of extending the Riccati equation approach to multi-dimensional structures. For the particular case of a 2-D model, the necessity of also considering the vertical impedances, or vertical admittances, for a complete characterization of the 2-D medium in terms of MT transfer functions, (12) and (13), follows immediately from the analogy with the 1-D case (11).

Acknowledgments. The financial support provided by the Grant Agency of the Czech Republic, through grants No. 205/99/0917 and No. 205/02/0381, is gratefully acknowledged. Additional support was also furnished by the Ministry of Education, Youth and Sports of the Czech Republic under the contract ME185 within the Czech-Japanese Cooperation in R\&D initiative. Moreover, we thank the reviewers of the first version of this paper, Mark Everett and Peter Weidelt, for their helpful and valuable comments and suggestions for improving the original manuscript.

\section{References}

Berdichevsky, M. N. and M. S. Zhdanov, Advanced Theory of Deep Geomagnetic Sounding, 408 pp., Elsevier, Amsterdam, 1984.

Chlamtac, M. and F. Abramovici, The electromagnetic fields of a horizontal dipole over a vertically inhomogeneous and anisotropic earth, Geophysics, 46, 904-915, 1981 .

Eckhardt, D. H., Geomagnetic induction in a concentrically stratified earth, J. Geophys. Res., 68, 6273-6278, 1963.

Everett, M. E. and S. Constable, Electric dipole fields over an anisotropic seafloor: theory and application to the structure of 40 Ma Pacific Ocean lithosphere, Geophys. J. Int., 136, 41-56, 1999.

Kováčiková, S. and J. Pek, Generalized Riccati equatios for 1-D magnetotelluric impedances over anisotropic conductors, Part I: Plane wave field model, Earth Planets Space, 54, this issue, 473-482, 2002.

Li, X. B. and L. B. Pedersen, Controlled-source tensor magnetotelluric responses of a layered earth with azimuthal anisotropy, Geophys. J. Int., 111, 91-103, 1992.

Xiong, Z., Electromagnetic fields of electric dipoles embedded in a stratified anisotropic earth, Geophysics, 54, 1643-1646, 1989.

Yin, C. C. and P. Weidelt, Geoelectrical fields in a layered earth with arbitrary anisotropy, Geophysics, 64, 426-434, 1999.

S. Kováčiková and J. Pek (e-mail: jpk@ig.cas.cz) 